\title{
Algebraic Modeling of the Turbulent Heat Fluxes Using the Elliptic Blending Approach-Application to Forced and Mixed Convection Regimes
}

\author{
Frederic Dehoux • Yannick Lecocq • \\ Sofiane Benhamadouche • Remi Manceau • \\ Laurent-Emmanuel Brizzi
}

Received: 6 December 2010 / Accepted: 18 August 2011

(C) Springer Science+Business Media B.V. 2011

\begin{abstract}
The present paper focuses on the application of the elliptic blending approach to the modeling of turbulent heat fluxes, in order to account for the influence of solid boundaries. The analytical justification of the extension to the temperature-pressure gradient correlation term of this approach, originally applied to the velocity-pressure gradient, is given. The assumption of weak equilibrium enables the derivation of two new algebraic flux models valid down to the wall. It is shown, with both a priori tests and computations in forced and mixed convection regimes, that the predictions of the streamwise heat-flux and the temperature variance are significantly improved by the use of elliptic blending. A particular attention is devoted to the issue of the modeling of the correlation length scale involved in the elliptic blending for the heat fluxes, which is shown to have a significant influence on the predictions.
\end{abstract}

Keywords Turbulent heat fluxes • Elliptic blending • Thermal length scale • Forced and mixed convection

\section{Introduction}

Many industrial applications, in particular in the field of energy production, are still treated with RANS eddy-viscosity models and the Simple Gradient Diffusion Hypothesis (SGDH), based on a turbulent Prandtl number, to model the turbulent heat fluxes in the Reynolds-averaged temperature equation. These simple models

F. Dehoux $\cdot$ Y. Lecocq $\cdot$ S. Benhamadouche $(\bowtie)$ EDF R\&D MFEE Department, Chatou, France e-mail: sofiane.benhamadouche@edf.fr

R. Manceau · L.-E. Brizzi

Institute Pprime, Department, Fluid flow, Heat Transfer and Combustion, CNRS-University of Poitiers-ENSMA, Poitiers, France 
have several limitations, either for the prediction of the dynamic of the flow or for the estimation of the Nusselt number distribution. This can be due to the inherent complexity of the dynamics of the flow, e.g., in impingement or swirling regions, as well as to complex coupling mechanisms, such as buoyancy effects.

The present article focuses on the so-called low-Reynolds number modeling for both the Reynolds stresses and the turbulent heat fluxes. In order to avoid the use of damping functions to replicate the effect of the presence of solid boundaries, based on a non-dimensional distance to the wall and/or a local turbulent Reynolds number, Durbin [6] introduced the Elliptic Relaxation approach. A simplified version, the Elliptic Blending approach, was proposed by Manceau and Hanjalić [24, 25], leading to the so-called Elliptic Blending Reynolds-Stress Model (EB-RSM), which was successfully applied to several configurations, in particular in isothermal and forced convection flows, in the context of RANS [4, 11, 13, 16, 29, 31, 32, 35-37], as well as, recently, hybrid RANS/LES $[9,10]$ (see also the review articles [8, 12]). This model is used in the present article to model the Reynolds stresses.

The modeling of the turbulent heat fluxes has attracted less efforts from the research community. As a consequence, several industrial computations are carried out with sophisticated Reynolds Stress Models but still use a Simple Gradient Diffusion Hypothesis for the heat fluxes, which is not satisfactory in mixed and natural convection regimes (e.g., see Kenjereš et al. [19]). The Generalized Gradient Diffusion Hypothesis (GGDH) [17] accounts for the influence of the anisotropy of the Reynolds stresses on turbulent heat fluxes, but is not sufficiently general to correctly represent buoyancy effects [14]. To overcome this problem, Algebraic Flux Models (AFMs) [5, 15, 20, 22] preserves the different production mechanisms arising in the exact transport equation for the turbulent heat fluxes.

However, in the existing AFMs, the effects of the walls are not taken into account. Similarly to the case of the Reynolds stresses mentioned above, in order to avoid the use of damping functions, the present paper aims at introducing the elliptic blending approach in algebraic flux models. Such models can be derived from the model recently proposed by Shin et al. [30], based on differential equations for the turbulent heat fluxes (EB-DFM, Elliptic Blending-Differential Flux Model). Therefore, the present paper aims at

- providing an in-depth justification for using elliptic relaxation/blending for the turbulent heat fluxes, based on a theoretical derivation using Green's formalism and an analysis of DNS budgets,

- investigating the validity of the simplifying hypothesis used in Shin et al. [30], which consists in using the same length scales in the elliptic blending equations for the Reynolds stresses and the turbulent heat fluxes,

- deriving Elliptic Blending-Algebraic Flux Models from the Elliptic BlendingDifferential Flux Model and validating them in forced and mixed convection cases.

\section{The Elliptic Blending Approach}

In this section, the main ideas leading to the elliptic blending approach for the velocity-pressure gradient correlation in the Reynolds stress transport equation are first recalled and its validity in cases with buoyancy is investigated. Finally, the 
extension of the approach to the modeling of the temperature pressure-gradient correlation term that enters the turbulent heat fluxes equations is presented.

2.1 Modeling the effect of the wall on the Reynolds-stresses

In a Reynolds Stress Model (RSM), in order to replicate the blocking of the wall-normal fluctuations, it can be shown, using a simple model for the two-point correlations and Green's formalism [7, 26], that the elliptic relaxation model

$$
\left(\phi_{i j}^{*}-\varepsilon_{i j}\right)-L^{2} \nabla^{2}\left(\phi_{i j}^{*}-\varepsilon_{i j}\right)=\left(\phi_{i j}^{h}-\varepsilon_{i j}^{h}\right)
$$

can be used for the difference between the velocity-pressure-gradient correlation term $\phi_{i j}^{*}$ and dissipation term $\varepsilon_{i j}$. The right hand side, $\phi_{i j}^{h}-\varepsilon_{i j}^{h}$, can be modeled using any available high-Reynolds number model. The main feature of this model is the satisfaction of the asymptotic, near-wall balance

$$
\phi_{i j}^{*}-\varepsilon_{i j}=-D_{i j}^{v}
$$

where $D_{i j}^{v}$ denotes the molecular diffusion tensors.

In order to avoid the resolution of six, strongly coupled and numerically unstable differential equations, a simplified version, the Elliptic Blending-RSM, was proposed by Manceau and Hanjalić [25] and improved by Manceau [24], based on a blending of any standard model for $\phi_{i j}^{h}-\varepsilon_{i j}^{h}$, valid far from the wall, and a near-wall model for $\phi_{i j}^{w}-\varepsilon_{i j}^{w}$ that satisfies the asymptotic balance (Eq. 2) in the near-wall region,

$$
\phi_{i j}^{*}-\varepsilon_{i j}=\left(1-\alpha^{3}\right)\left(\phi_{i j}^{w}-\varepsilon_{i j}^{w}\right)+\alpha^{3}\left(\phi_{i j}^{h}-\varepsilon_{i j}^{h}\right) .
$$

In order to preserve the non-local character of the blocking effect, similarly to Durbin's elliptic relaxation model, the blending function $\alpha$ is obtained from an elliptic relaxation equation

$$
\alpha-L^{2} \nabla^{2} \alpha=1 \quad \text { with }\left.\quad \alpha\right|_{w}=0,
$$

and goes from 0 at the wall to 1 far from the wall.

If Rotta's model [28] $\left(\varepsilon_{i j}^{w}=\overline{u_{i}^{\prime} u_{j}^{\prime}} / k \varepsilon\right)$ is used, the asymptotic analysis in the vicinity of the wall shows that the following model for $\phi_{i j}^{w}$ is consistent with the wall limits

$$
\phi_{i j}^{w}=-5 \frac{\varepsilon}{k}\left[\overline{u_{i}^{\prime} u_{k}^{\prime}} n_{j} n_{k}+\overline{u_{j}^{\prime} u_{k}^{\prime}} n_{i} n_{k}-\frac{1}{2} \overline{u_{k}^{\prime} u_{l}^{\prime}} n_{k} n_{l}\left(n_{i} n_{j}+\delta_{i j}\right)\right],
$$

where $\mathbf{n}$ is a unit vector

$$
\mathbf{n}=\nabla \alpha /\|\nabla \alpha\|,
$$

providing a generalized wall-normal direction. Far from the wall, the SSG model (Speziale et al. [33]) is used for the redistribution term and an isotropic model for the dissipation tensor $\left(\varepsilon_{i j}^{h}=2 / 3 \varepsilon \delta_{i j}\right)$.

In cases where buoyancy is present, the issue of the validity of the EB-RSM, which is based on the satisfaction of the near-wall balance (Eq. 2), arises. Close to a wall located at $y=0$, Taylor-series expansions lead to the behaviors given in Table 1 for the Reynolds stress budgets when the temperature fluctuation is zero at the wall, which is the case in the present article. It can be seen that the production 
Table 1 Asymptotic behaviors of the terms of the Reynolds stress budget in buoyancy affected flows

\begin{tabular}{lllllll}
\hline & $D_{i j}^{v}$ & $\phi_{i j}^{*}$ & $\varepsilon_{i j}$ & $P_{i j}$ & $D_{i j}^{T}$ & $G_{i j}$ \\
\hline$\overline{\frac{u^{\prime 2}}{v^{\prime 2}}}$ & $\mathcal{O}(1)$ & $\mathcal{O}(y)$ & $\mathcal{O}(1)$ & $\mathcal{O}\left(y^{3}\right)$ & $\mathcal{O}\left(y^{3}\right)$ & $\mathcal{O}\left(y^{2}\right)$ \\
$\overline{w^{\prime 2}}$ & $\mathcal{O}\left(y^{2}\right)$ & $\mathcal{O}\left(y^{2}\right)$ & $\mathcal{O}\left(y^{2}\right)$ & $\mathcal{O}\left(y^{5}\right)$ & $\mathcal{O}\left(y^{5}\right)$ & $\mathcal{O}\left(y^{3}\right)$ \\
$\overline{u^{\prime} v^{\prime}}$ & $\mathcal{O}(1)$ & $\mathcal{O}(y)$ & $\mathcal{O}(1)$ & $\mathcal{O}\left(y^{3}\right)$ & $\mathcal{O}\left(y^{3}\right)$ & $\mathcal{O}\left(y^{2}\right)$ \\
$\overline{u^{\prime} w^{\prime}}$ & $\mathcal{O}(y)$ & $\mathcal{O}(y)$ & $\mathcal{O}(y)$ & $\mathcal{O}\left(y^{4}\right)$ & $\mathcal{O}\left(y^{4}\right)$ & $\mathcal{O}\left(y^{2}\right)$ \\
$\overline{v^{\prime} w^{\prime}}$ & $\mathcal{O}(1)$ & $\mathcal{O}(y)$ & $\mathcal{O}(1)$ & $\mathcal{O}\left(y^{3}\right)$ & $\mathcal{O}\left(y^{3}\right)$ & $\mathcal{O}\left(y^{2}\right)$ \\
\hline
\end{tabular}

due to buoyancy $G_{i j}=-\beta\left(\overline{u_{i}^{\prime} \theta^{\prime}} g_{j}+\overline{u_{j}^{\prime} \theta^{\prime}} g_{i}\right)$ does not affect the balance given by Eq. 2, and, in particular, that $G_{i j} / \phi_{i j}^{*}=\mathcal{O}(y)$ for all $i$ and $j$. It can be concluded that the EB-RSM does not require any further modification while introducing buoyancy terms. However, a modification of the transition from the near-wall behavior to the far-from-the-wall behavior can be expected, which would question the validity of Eq. 4. Nevertheless, using the DNS data of Kasagi and Nishimura [18], in the mixed convection case of a vertical channel with a pressure gradient and a temperature difference between the two walls (a more detailed description of this case is provided in Section 4), it can be seen in Fig. 1 that the blending function $\alpha$ is only marginally modified by buoyancy. It is worth recalling here that the model is formulated to reproduce the wall-blocking effect, i.e., to specifically damp the redistribution of energy toward the wall-normal fluctuations. Therefore, the evaluation of the blending parameter $\alpha$ and the length scale $L$ to be reproduced by the model can be performed by using the components $\phi_{22}^{*}$ and $\varepsilon_{22}$ of the DNS databases (a priori evaluation), by computing

$$
\alpha^{3}=\frac{\left(\phi_{22}^{*}-\varepsilon_{22}\right)-\left(\phi_{22}^{w}-\varepsilon_{22}^{w}\right)}{\left(\phi_{22}^{h}-\varepsilon_{22}^{h}\right)-\left(\phi_{22}^{w}-\varepsilon_{22}^{w}\right)},
$$

Similar to the isothermal case, it can be seen that $\alpha$ varies between 0 and 1 , and even though it becomes slightly asymmetrical, the slope in the near-wall region (i.e., the

Fig. 1 Influence of buoyancy on $\alpha$ evaluated a priori by Eq. 6. DNS data from Kasagi and Nishimura [18]

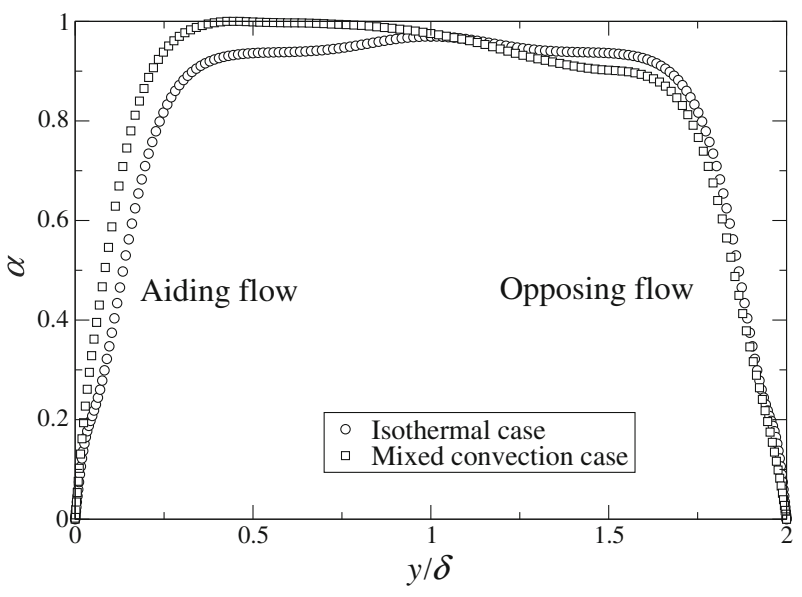


extent of the influence of the wall) is only weakly modified. This modification and the asymmetry can be explained (and thus reproduced) by the influence of buoyancy on turbulence, and thus on the length scale $L=C_{L} \max \left(k^{3 / 2} / \varepsilon, C_{\eta} v^{3 / 4} / \varepsilon^{1 / 4}\right)$. Therefore, it is considered that the EB-RSM does not require any modification when buoyancy effects are present.

\subsection{Extension to the modeling of the effect of the wall on the heat fluxes}

As it is the case for the redistribution term in the Reynolds stress transport equations, a model for the scrambling term in the transport equation for the turbulent heat fluxes can be derived from the Poisson equation of the fluctuating pressure.

The momentum and continuity equations are written in the RANS form with a Boussinesq approximation

$$
\begin{gathered}
\frac{\partial \overline{u_{j}}}{\partial x_{j}}=0 \\
\frac{\partial \overline{u_{i}}}{\partial t}+\frac{\partial\left(\overline{u_{i}} \overline{u_{j}}\right)}{\partial x_{j}}=-\frac{1}{\rho_{0}} \frac{\partial \bar{p}}{\partial x_{i}}+v \frac{\partial^{2} \overline{u_{i}}}{\partial x_{j} \partial x_{j}}-\frac{\partial R_{i j}}{\partial x_{j}}+\frac{\bar{\rho}}{\rho_{0}} g_{i}
\end{gathered}
$$

where $u_{i}$ are the velocity components, $p$ the pressure, $R_{i j}$ the Reynolds stresses, $v$ the kinematic viscosity, $\rho$ the (temperature-dependent) density and $\rho_{0}$ a reference density.

The mean temperature equation is given by

$$
\frac{\partial \bar{\theta}}{\partial t}+\frac{\partial\left(\overline{u_{j}} \bar{\theta}\right)}{\partial x_{j}}=\frac{\partial}{\partial x_{j}}\left(\frac{v}{\operatorname{Pr}} \frac{\partial \bar{\theta}}{\partial x_{j}}-\overline{u_{j}^{\prime} \theta^{\prime}}\right)
$$

A Poisson equation can be obtained for the gradient of the fluctuating pressure

$$
\nabla^{2} \frac{\partial p^{\prime}}{\partial x_{k}}=-\rho_{0} \frac{\partial}{\partial x_{k}}\left(2 \frac{\partial \overline{u_{i}}}{\partial x_{j}} \frac{\partial u_{j}^{\prime}}{\partial x_{i}}+\frac{\partial u_{i}^{\prime}}{\partial x_{j}} \frac{\partial u_{j}^{\prime}}{\partial x_{i}}-\overline{\frac{\partial u_{i}^{\prime}}{\partial x_{j}} \frac{\partial u_{j}^{\prime}}{\partial x_{i}}}-\frac{1}{\rho_{0}} g_{l} \frac{\partial \rho^{\prime}}{\partial x_{l}}\right)
$$

Using the Green function of the domain and assuming that $\frac{\partial p^{\prime}}{\partial x_{k}}$ satisfies a Neuman boundary condition (see Manceau et al. [26]), the solution of Eq. 10 can be obtained in the integral form

$$
\frac{\partial p^{\prime}}{\partial x_{k}}(x)=\int \nabla^{2} \frac{\partial p^{\prime}}{\partial x_{k}}(y) G(x, y) d y
$$

The scrambling term (temperature pressure-gradient one-point correlation) that arises in the turbulent heat flux transport equation is

$$
\phi_{\theta i}^{*}=-\frac{1}{\rho_{0}} \overline{\theta^{\prime}} \overline{\frac{\partial p^{\prime}}{\partial x_{i}}},
$$

such that

$$
\rho_{0} \phi_{\theta i}^{*}=\int \Psi_{\theta i}(x, y) G(x, y) d y
$$


where $\Psi_{\theta i}$ is the two-point correlation between the temperature and the Laplacian of the pressure gradient:

$$
\Psi_{\theta i}(x, y)=\overline{-\theta^{\prime}(x) \nabla^{2} \frac{\partial p^{\prime}}{\partial x_{k}}(y)}
$$

Similarly to what was proposed by Durbin [6] and justified by Manceau et al. [26] for the velocity-pressure gradient two-point correlation, an exponential decay is assumed

$$
\Psi_{\theta i}(x, y)=\Psi_{\theta i}(y, y) e^{-\frac{r}{L_{\theta}}}
$$

where $r=\|x-y\|$, and the correlation length scale $L_{\theta}$ is to be determined. Using this assumption, the scrambling term reads

$$
\rho_{0} \phi_{\theta i}^{*}=\frac{1}{4 \pi} \int \Psi_{\theta i}(y, y) \frac{\exp \left(-r / L_{\theta}\right)}{r} d y .
$$

Since $\frac{1}{4 \pi} \frac{\exp \left(-r / L_{\theta}\right)}{r}$ is the Green's function associated to the operator $-\nabla^{2}+\frac{1}{L_{\theta}^{2}} I d$, an elliptic relaxation equation is obtained for $\phi_{\theta i}^{*}$

$$
\phi_{\theta i}^{*}-L_{\theta}^{2} \nabla^{2} \phi_{\theta i}^{*}=-\frac{1}{\rho_{0}} L_{\theta}^{2} \overline{\theta^{\prime}} \overline{\frac{\partial p^{\prime}}{\partial x_{i}}} .
$$

In a homogeneous flow, this equation reduces to

$$
\phi_{\theta i}^{*}=-\frac{1}{\rho_{0}} L_{\theta}^{2} \overline{\theta^{\prime}} \overline{\frac{\partial p^{\prime}}{\partial x_{i}}}
$$

Consequently, the right hand side of the equation can be modeled using any quasihomogeneous model, such that the elliptic relaxation provides the appropriate nearwall treatment for standard high Reynolds models

$$
\phi_{\theta i}^{*}-L_{\theta}^{2} \nabla^{2} \phi_{\theta i}^{*}=\phi_{\theta i}^{h} .
$$

The above derivation justifies the use of the elliptic relaxation approach to the turbulent heat fluxes, as proposed by Shin et al. [30] based on a simple analogy with the Reynolds stresses.

Instead of solving three equations for individual components of the heat fluxes and following Manceau and Hanjalić [25], Shin et al. [30] extended the elliptic blending approach to the heat fluxes, leading to the Elliptic Blending-Differential Flux Model (EB-DFM)

$$
\phi_{\theta i}^{*}-\varepsilon_{\theta i}=\left(1-\alpha_{\theta}^{3}\right)\left(\phi_{\theta i}^{w}-\varepsilon_{\theta i}^{w}\right)+\alpha_{\theta}^{3}\left(\phi_{\theta i}^{h}-\varepsilon_{\theta i}^{h}\right) .
$$

The parameter $\alpha_{\theta}$, used to blend the near-wall and far-from-the-wall forms of the heat fluxes model is obtained from the elliptic relaxation equation

$$
\alpha_{\theta}-L_{\theta}^{2} \nabla^{2} \alpha_{\theta}=1
$$

with the boundary condition $\alpha_{\theta}=0$ at the wall. This equation ensures that $\alpha_{\theta} \rightarrow 1$ far from the wall. Contrary to Shin et al. [30], it is not assumed here that the length scale $L_{\theta}$ is equal to the dynamic length scale $L$ used in Eq. 4, i.e., for the difference 
$\phi_{i j}^{*}-\varepsilon_{i j}$ in the Reynolds-stress transport equations, which is, following Durbin [6], modeled by

$$
L=C_{L} \max \left(\frac{k^{3 / 2}}{\varepsilon}, C_{\eta} \frac{v^{3 / 4}}{\varepsilon^{1 / 4}}\right)
$$

with $C_{L}=0.125$ and $C_{\eta}=80$.

Table 2 gives the asymptotic behavior of the different terms on the r.h.s. of the transport equations for the turbulent heat fluxes for zero temperature fluctuations at the wall (see Eq. 24). Similar to the case of the Reynolds stresses, the near-wall budget of the wall normal heat flux reduces, at leading order, to a balance between scrambling, dissipation and molecular diffusion, $\phi_{\theta 2}^{*}-\varepsilon_{\theta 2}+D_{\theta 2}^{v}=0$. In order to ensure the correct asymptotic behavior at the wall of $\phi_{\theta i}^{w}-\varepsilon_{\theta i}^{w}$, Shin et al. [30] showed that the near-wall terms can be modeled as

$$
\phi_{\theta i}^{w}=-\left[1+\frac{1}{2}\left(1+\frac{1}{\operatorname{Pr}}\right)\right] \frac{\varepsilon}{k} \overline{u_{j}^{\prime} \theta^{\prime}} n_{i} n_{j}
$$

and

$$
\varepsilon_{\theta i}^{w}=\frac{1}{2}\left(1+\frac{1}{\operatorname{Pr}}\right) \frac{\varepsilon}{k} \overline{u_{i}^{\prime} \theta^{\prime}} .
$$

Note that this model does not satisfy the individual asymptotic behaviors of $\phi_{\theta i}^{w}$ and $\varepsilon_{\theta i}^{w}$, but only of their difference. This problem can be simply overcome by using

$$
\phi_{\theta i}^{w}=-\frac{\varepsilon}{k} \overline{u_{j}^{\prime} \theta^{\prime}} n_{i} n_{j}
$$

and

$$
\varepsilon_{\theta i}^{w}=\frac{1}{2}\left(1+\frac{1}{\operatorname{Pr}}\right) \frac{\varepsilon}{k}\left(\overline{u_{i}^{\prime} \theta^{\prime}}+\overline{u_{j}^{\prime} \theta^{\prime}} n_{i} n_{j}\right),
$$

which is, in practice, strictly equivalent to the previous model, since the difference $\phi_{\theta i}^{w}$ and $\varepsilon_{\theta i}^{w}$ is the same. Far from the wall, the model 13 tend to the high-Reynolds number model $\phi_{\theta i}^{h}-\varepsilon_{\theta i}^{h}$. Shin et al. [30] proposed to use the standard linear model [23],

$$
\phi_{\theta i}^{h}=-C_{\theta 1} \frac{\varepsilon}{k} \overline{u_{i}^{\prime} \theta^{\prime}}+C_{\theta 2} \overline{u_{j}^{\prime} \theta^{\prime}} \frac{\partial \overline{u_{i}}}{\partial x_{j}}+C_{\theta 2}^{\prime} \overline{u_{i}^{\prime} u_{j}^{\prime}} \frac{\partial \bar{\theta}}{\partial x_{j}}+C_{\theta 3} g_{i} \beta \overline{\theta^{\prime 2}}
$$

and

$$
\varepsilon_{\theta i}^{h}=0
$$

Table 2 Asymptotic behaviors of the terms of the heat fluxes budget (Shin et al. [30])

\begin{tabular}{lllllll}
\hline & $P_{\theta i}^{U}+P_{\theta i}^{T}$ & $G_{\theta i}$ & $\phi_{\theta i}^{*}$ & $D_{\theta i}^{v}$ & $-\varepsilon_{\theta i}$ & $D_{\theta i}^{t}$ \\
\hline$\overline{u^{\prime} \theta^{\prime}}$ & $\mathcal{O}\left(y^{3}\right)$ & $\mathcal{O}\left(y^{2}\right)$ & $\mathcal{O}(y)$ & $\mathcal{O}(1)$ & $\mathcal{O}(1)$ & $\mathcal{O}\left(y^{3}\right)$ \\
$\overline{v^{\prime} \theta^{\prime}}$ & $\mathcal{O}\left(y^{4}\right)$ & $\mathcal{O}\left(y^{2}\right)$ & $\mathcal{O}(y)$ & $\mathcal{O}(y)$ & $\mathcal{O}(y)$ & $\mathcal{O}\left(y^{4}\right)$ \\
$\overline{w^{\prime} \theta^{\prime}}$ & $\mathcal{O}\left(y^{3}\right)$ & $\mathcal{O}\left(y^{2}\right)$ & $\mathcal{O}(y)$ & $\mathcal{O}(1)$ & $\mathcal{O}(1)$ & $\mathcal{O}\left(y^{3}\right)$ \\
\hline
\end{tabular}

$y$ denotes the distance to the wall 
In order to write equations as general as possible, the high-Reynolds number model $\phi_{\theta i}^{h}$ for the pressure scrambling term used here is the nonlinear model of Kenjereš et al. [19], consisting of the rapid part

$$
\phi_{\theta i}^{r}=C_{\theta 2} \overline{u_{j}^{\prime} \theta^{\prime}} \frac{\partial \overline{u_{i}}}{\partial x_{j}}+C_{\theta 2}^{\prime} \overline{u_{i}^{\prime} u_{j}^{\prime}} \frac{\partial \bar{\theta}}{\partial x_{j}}+C_{\theta 3} g_{i} \beta \overline{\theta^{\prime 2}}
$$

and the slow part

$$
\phi_{\theta i}^{s}=-C_{\theta 1} \frac{\varepsilon}{k}\left(\overline{u_{i}^{\prime} \theta^{\prime}}-C_{\theta 1}^{\prime} a_{i j} \overline{u_{j}^{\prime} \theta^{\prime}}\right)
$$

where $a_{i j}$ is the stress-anisotropy tensor, $a_{i j}=R_{i j} / k-2 / 3 \delta_{i j}$. The linear highReynolds number model (Eq. 20) is obviously a particular case of this model.

\section{Algebraic Modeling of the Heat Fluxes}

Although differential flux modeling is the appropriate level to represent the physical mechanisms driving the evolution of the turbulent heat fluxes, industrial application still rely on much simpler models. The aim of the present section is to make available an intermediate model based on the elliptic blending method to represent the nearwall effects, i.e., an algebraic flux model derived from the EB-DFM presented above. After recalling the method used to derive the standard AFM, this method is applied to the EB-DFM, leading to the Elliptic Blending-Algebraic Flux Model (EB-AFM). Similar to the case of GGDH, which can be written as a simplification of the AFM, a simple model, the EB-GGDH (Elliptic Blending-Generalized Gradient Diffusion Hypothesis), is readily obtained.

\subsection{The standard AFM}

Using the Boussinesq approximation, the transport equations for the turbulent heat fluxes can be written

$$
\begin{aligned}
& \underbrace{\frac{\mathrm{D} \overline{u_{i}^{\prime} \theta^{\prime}}}{\mathrm{D} t}}_{C_{\theta i}}=\underbrace{-\overline{u_{j}^{\prime} \theta^{\prime}} \frac{\partial \overline{u_{i}}}{\partial x_{j}}}_{P_{\theta i}^{U}}-\underbrace{-\overline{u_{i}^{\prime} u_{j}^{\prime}} \frac{\partial \bar{\theta}}{\partial x_{j}}}_{P_{\theta i}^{T}} \underbrace{-g_{i} \beta \overline{\theta^{\prime 2}}}_{G_{\theta i}}-\underbrace{\bar{\rho} \frac{\theta^{\prime}}{\rho} \frac{\partial p^{\prime}}{\partial x_{i}}}_{\phi_{\theta i}^{*}} \underbrace{(\lambda+v) \frac{\partial \theta^{\prime} \frac{\partial u_{i}^{\prime}}{\partial x_{j}} \frac{\partial x_{j}}{\partial x_{j}}}{(\lambda}}_{\varepsilon_{\theta i}} \\
& +\underbrace{\frac{\partial}{\partial x_{j}}\left(-\overline{\theta^{\prime} u_{i}^{\prime} u_{j}^{\prime}}\right)}_{D_{\theta i}^{t}}+\underbrace{\frac{\partial}{\partial x_{j}}\left(\lambda \overline{\frac{\partial \theta^{\prime}}{\partial x_{j}} u_{i}^{\prime}}+v \overline{\theta^{\prime} \frac{\partial u_{i}^{\prime}}{\partial x_{j}}}\right)}_{D_{\theta i}^{v}}
\end{aligned}
$$

where $C_{\theta i}, P_{\theta i}^{U}, P_{\theta i}^{T}, G_{\theta i}, \phi_{\theta i}^{*}, \varepsilon_{\theta i}, D_{\theta i}^{t}$ and $D_{\theta i}^{v}$ denote the material derivative, the production by the mean velocity gradient, by the mean temperature gradient, by buoyancy, the temperature-pressure gradient correlation, the dissipation, the turbulent and molecular diffusions, respectively.

Algebraic forms of the turbulent heat flux transport equation can be derived, similarly to the Reynolds stress transport equations (see, for example, [27]). 
As detailed by Hanjalić [14], algebraic models for the heat fluxes rely on the weak equilibrium assumptions for convection

$$
\frac{\mathrm{d} \overline{u_{i}^{\prime} \theta^{\prime}}}{\mathrm{d} t}=\frac{1}{2}\left(\frac{1}{k} \frac{\mathrm{d} k}{\mathrm{~d} t}+\frac{1}{\overline{\theta^{\prime 2}}} \frac{\mathrm{d} \overline{\theta^{\prime 2}}}{\mathrm{~d} t}\right) \overline{u_{i}^{\prime} \theta^{\prime}}
$$

and diffusion

$$
\mathcal{D}_{\theta i}=\frac{1}{2}\left(\frac{1}{k} \mathcal{D}_{k}+\frac{1}{\overline{\theta^{\prime 2}}} \mathcal{D}_{\overline{\theta^{\prime 2}}}\right) \overline{u_{i}^{\prime} \theta^{\prime}}
$$

where $\mathcal{D}_{\theta i}, \mathcal{D}_{k}$ and $\mathcal{D}_{\overline{\theta^{\prime 2}}}$ denote the total diffusion of $\overline{u_{i}^{\prime} \theta^{\prime}}, k$ and $\overline{\theta^{\prime 2}}$, respectively, which yields the turbulent heat flux algebraic equation

$$
P_{\theta i}+\phi_{\theta i}^{*}-\varepsilon_{\theta i}-\frac{\overline{u_{i}^{\prime} \theta^{\prime}}}{2 k}\left(P_{k}+G_{k}-\varepsilon\right)-\frac{\overline{u_{i}^{\prime} \theta^{\prime}}}{2 \overline{\theta^{\prime 2}}}\left(P_{\overline{\theta^{\prime 2}}}-\varepsilon \overline{\theta^{\prime 2}}\right)=0,
$$

where $P_{\theta i}=P_{\theta i}^{U}+P_{\theta i}^{T}+G_{\theta i}$.

If the production and the dissipation terms of both $k$ and $\overline{\theta^{\prime 2}}$ are assumed to be locally in balance, i.e., turbulence is assumed to be in local equilibrium,

$$
P_{k}+G_{k}=\varepsilon
$$

and

$$
P_{\overline{\theta^{\prime 2}}}=\varepsilon \overline{\theta^{\prime 2}},
$$

then Eq. 27 reduces to

$$
P_{\theta i}+\phi_{\theta i}^{*}-\varepsilon_{\theta i}=0 .
$$

Introducing the nonlinear model (Eqs. 22 and 23) for the scrambling term in this relation yields

$$
\begin{aligned}
C_{\theta 1} \frac{\varepsilon}{k} \overline{u_{i}^{\prime} \theta^{\prime}}= & \left(1-C_{\theta 2}\right) P_{\theta i}^{U}+\left(1-C_{\theta 2}^{\prime}\right) P_{\theta i}^{T} \\
& +\left(1-C_{\theta 3}\right) G_{\theta i}+C_{\theta 1} C_{\theta 1}^{\prime} \frac{\varepsilon}{k} a_{i j} \overline{u_{j}^{\prime} \theta^{\prime}}-\varepsilon_{\theta i}^{h}
\end{aligned}
$$

For Prandtl numbers not small compared to unity, temperature fluctuations at small scales can be considered isotropic [34], such that the dissipation term is assumed to be zero far from the wall $\left(\varepsilon_{\theta i}^{h}=0\right)$. Equation 24 thus reduces to the Non-Linear Algebraic Flux Model (NL-AFM), Kenjereš et al. [19],

$$
\overline{u_{i}^{\prime} \theta^{\prime}}=-C_{\theta} \frac{k}{\varepsilon}\left[\zeta \overline{u_{i}^{\prime} u_{j}^{\prime}} \frac{\partial \bar{\theta}}{\partial x_{j}}+\xi \overline{u_{j}^{\prime} \theta^{\prime}} \frac{\partial \overline{u_{i}}}{\partial x_{j}}+\eta \beta g_{i} \overline{\theta^{\prime 2}}-\chi \frac{\varepsilon}{k} a_{i j} \overline{u_{j}^{\prime} \theta^{\prime}}\right]
$$

where $C_{\theta}=C_{\theta}^{\prime} / C_{\theta 1}, \zeta=1-C_{\theta 2}^{\prime}, \xi=1-C_{\theta 2}, \eta=1-C_{\theta 3}$ and $\chi=C_{\theta 1} C_{\theta 1}^{\prime}$. Two simplifications of this model will be utilized in the following: the linear Algebraic Flux Model (AFM, $\chi=0$ ) and the Generalized Gradient Diffusion Hypothesis (GGDH, $\xi=\eta=\chi=0)$. Throughout the present paper, the coefficients that are generally recommended [14], $C_{\theta 1}=3.0, C_{\theta 2}^{\prime}=0, C_{\theta 2}=C_{\theta 3}=0.55$, are used . Note that the coefficient $C_{\theta}$, which should be equal to $1 / C_{\theta 1}$, is modified by the introduction of 
the coefficient $C_{\theta}^{\prime}$; indeed, the assumptions used in the algebraic methodology make necessary the introduction of this recalibration coefficient.

\subsection{Elliptic blending-algebraic flux model}

The model for the scrambling term given by Eqs. 22 and 23, and consequently the AFM, does not account for the effect of the wall on turbulence. In order to overcome this limitation, the main proposal of the present paper is to apply the elliptic blending strategy to the heat fluxes in order to derive the Elliptic Blending-Algebraic Flux Model (EB-AFM).

The same derivation as the one done in the Section 3.1 for the high-Reynolds number model but now applied to the EB-DFM described in Section 2.2 then leads to

$$
\begin{gathered}
P_{\theta i}^{U}+P_{\theta i}^{T}+G_{\theta i}-\frac{\overline{u_{i}^{\prime} \theta^{\prime}}}{2 k}\left(P_{k}+G_{k}\right)-\overline{\frac{u_{i}^{\prime} \theta^{\prime}}{\overline{\theta^{\prime 2}}}} P_{\overline{\theta^{\prime 2}}} \\
-\left(1-\alpha_{\theta}^{3}\right) \frac{\varepsilon}{k} \overline{u_{j}^{\prime} \theta^{\prime}} n_{i} n_{j}-\alpha_{\theta}^{3} C_{\theta 1} \frac{\varepsilon}{k} \overline{u_{i}^{\prime} \theta^{\prime}}+\alpha_{\theta}^{3} C_{\theta 1} C_{\theta 1}^{\prime} \frac{\varepsilon}{k} a_{i j} \overline{u_{j} \theta}-\alpha_{\theta}^{3} C_{\theta 2} P_{\theta i}^{U}-\alpha_{\theta}^{3} C_{\theta 2}^{\prime} P_{\theta i}^{T} \\
-\alpha_{\theta}^{3} C_{\theta 3} G_{i}-\left(1-\alpha_{\theta}^{3}\right) C_{\varepsilon} \frac{\varepsilon}{k}\left(\overline{u_{i}^{\prime} \theta^{\prime}}+\overline{u_{j}^{\prime} \theta^{\prime}} n_{i} n_{j}\right)-\alpha_{\theta}^{3} \varepsilon_{\theta i}^{h}+\overline{u_{i}^{\prime} \theta^{\prime}}\left(\frac{\varepsilon}{2 k}+\frac{\varepsilon \overline{\theta^{\prime 2}}}{2 \overline{\theta^{\prime 2}}}\right)=0,
\end{gathered}
$$

such that the algebraic model now reads, assuming equilibrium (Eqs. 27 and 28),

$$
\overline{u_{i}^{\prime} \theta^{\prime}}=\frac{\xi P_{\theta i}^{U}+\zeta P_{\theta i}^{T}+\eta G_{\theta i}-\gamma \frac{\varepsilon}{k} \overline{u_{j}^{\prime} \theta^{\prime}} n_{i} n_{j}+\chi \frac{\varepsilon}{k} a_{i j} \overline{u_{j}^{\prime} \theta^{\prime}}-\alpha_{\theta}^{3} \varepsilon_{i \theta}^{h}}{\left[\alpha_{\theta}^{3} C_{\theta 1}+\left(1-\alpha_{\theta}^{3}\right) C_{\varepsilon}\right] \frac{\varepsilon}{k}}
$$

where $\xi=\left(1-\alpha_{\theta}^{3} C_{\theta 2}\right), \zeta=\left(1-\alpha_{\theta}^{3} C_{\theta 2}^{\prime}\right), \chi=\alpha_{\theta}^{3} C_{\theta 1} C_{\theta 1}^{\prime}, \eta=\left(1-\alpha_{\theta}^{3} C_{\theta 3}\right), \gamma=(1-$ $\left.\alpha_{\theta}^{3}\right)\left(1+C_{\varepsilon}\right), C_{\varepsilon}=\frac{1}{2}(1+1 / P r)$. Assuming that $\varepsilon_{i \theta}^{h}=0$, this relation reduces to

$$
\overline{u_{i}^{\prime} \theta^{\prime}}=-C_{\theta} \frac{k}{\varepsilon}\left[\zeta \overline{u_{i}^{\prime} u_{j}^{\prime}} \frac{\partial \bar{\theta}}{\partial x_{j}}+\xi \overline{u_{j}^{\prime} \theta^{\prime}} \frac{\partial \overline{u_{i}}}{\partial x_{j}}+\eta \beta g_{i} \overline{\theta^{\prime 2}}-\chi \frac{\varepsilon}{k} a_{i j} \overline{u_{j}^{\prime} \theta^{\prime}}+\gamma \frac{\varepsilon}{k} \overline{u_{j}^{\prime} \theta^{\prime}} n_{i} n_{j}\right],
$$

where

$$
C_{\theta}=\frac{C_{\theta}^{\prime}}{\alpha_{\theta}^{3} C_{\theta 1}+\left(1-\alpha_{\theta}^{3}\right) C_{\varepsilon}}
$$

This model only differs from Eq. 31 by the additional term $\gamma \frac{\varepsilon}{k} \overline{u_{j}^{\prime} \theta^{\prime}} n_{i} n_{j}$, which sensitizes the model to the orientation of the wall, and, above all, by the fact that the coefficients $\xi, \zeta, \chi, \eta$ and $C_{\theta}$ are now dependent on the blending function $\alpha_{\theta}$. It is easy to see that in regions far from the wall, where $\alpha_{\theta} \rightarrow 1$, the influence of the elliptic blending method vanishes and the model (Eq. 31) is recovered.

In the following, the usual constants of the linear high-Reynolds number model are used: $C_{\theta 1}=3, C_{\theta 2}=0.55, C_{\theta 2}^{\prime}=0, C_{\theta 3}=0.55$ and $C_{\theta 1}^{\prime}=0$. Note that the elliptic blending method does not introduce any new coefficient. 
Moreover, the model can be further simplified by using $\zeta=1$ and $\xi=\eta=\chi=0$, which yields the EB-GGDH model

$$
\overline{u_{i}^{\prime} \theta^{\prime}}=-C_{\theta} \frac{k}{\varepsilon}\left[\overline{u_{i}^{\prime} u_{j}^{\prime}} \frac{\partial \bar{\theta}}{\partial x_{j}}+\gamma \frac{\varepsilon}{k} \overline{u_{j}^{\prime} \theta^{\prime}} n_{i} n_{j}\right] .
$$

\subsection{Equation for the temperature variance}

The transport equation for the temperature variance $\overline{\theta^{\prime 2}}$, involved in Eq. 34, is written as

$$
\frac{\partial \overline{\theta^{\prime 2}}}{\partial t}+\frac{\partial\left(\overline{u_{j}} \overline{\theta^{\prime 2}}\right)}{\partial x_{j}}=\frac{\partial}{\partial x_{k}}\left[\left(\frac{v}{\operatorname{Pr}} \delta_{k l}+C_{\theta \theta} \rho \overline{u_{k}^{\prime} u_{l}^{\prime}} T\right) \frac{\partial \overline{\theta^{\prime 2}}}{\partial x_{l}}\right]+P_{\theta \theta}-\varepsilon_{\theta \theta}
$$

In this equation, the production term, equal to

$$
P_{\theta \theta}=-2 \overline{u_{i}^{\prime} \theta^{\prime}} \frac{\partial \bar{\theta}}{\partial x_{i}}
$$

does not require modeling, and the dissipation rate $\varepsilon_{\theta \theta}$ is obtained via the ratio $R$ of thermal to mechanical time scales

$$
R=\overline{\theta^{\prime 2}} \frac{\varepsilon}{\varepsilon_{\theta \theta}} \bar{k} .
$$

For large turbulent Peclet numbers, which can be considered valid far from the wall, for fluids with not too small Prandtl numbers, assuming a constant thermal-tomechanical time-scale ratio $R=R^{h}$, where the superscript $h$ denotes homogeneous or quasi-homogeneous, is a usual practice [14]. In the present case, where the Prandtl number is close to unity, the widely admitted value $R^{h}=0.5$ is used.

However, in the near-wall region, this assumption fails and, in particular, $R$ approaches the Prandtl number at the wall. In order to account for this limit, in the framework of the elliptic blending approach, $R$ can be modeled as

$$
R=\alpha_{\theta}^{3} R^{h}+\left(1-\alpha_{\theta}^{3}\right) \operatorname{Pr}
$$

The DNS database of Abe and Kawamura [1], in which the ratio $R$ is available, enables the evaluation of the performance of this simple model in forced convection regime. The flow between two parallel flat plates is driven by an imposed pressure gradient (see Fig. 3), and a constant wall heat flux $\dot{q}_{w}$ is imposed. Buoyancy terms are neglected, such that temperature is a passive scalar. The two parameters of the flow are the friction Reynolds number $R e_{\tau}$ and the Prandtl number $\operatorname{Pr}=0.71$. Figure 2 shows the time-scale ratio $R$ obtained with Eq. 38, for several value of the length scale used in the equation of $\alpha_{\theta}^{3}$ (see Section 4 for the discussion about this length scale). In this a priori procedure, in order to obtain $\alpha_{\theta}$, Eq. 14 is first solved, with the mechanical length scale $L$ evaluated using DNS data and $L_{\theta}$ taken proportional to $L$. It can be seen that using the simple model (Eq. 38) yields a significant improvement of the prediction of $R$ in the near-wall region, although the complex shape below $y^{+}=50$ is not reproduced. Therefore, Eq. 38 will be used in the computations presented in Section 5 for the EB-AFM and EB-GGDH models. 
Fig. 2 Thermal-to-mechanical time-scale ratio $R$ obtained from Eq. 38. A priori test in forced convection, $R e_{\tau}=640$ ) [1]

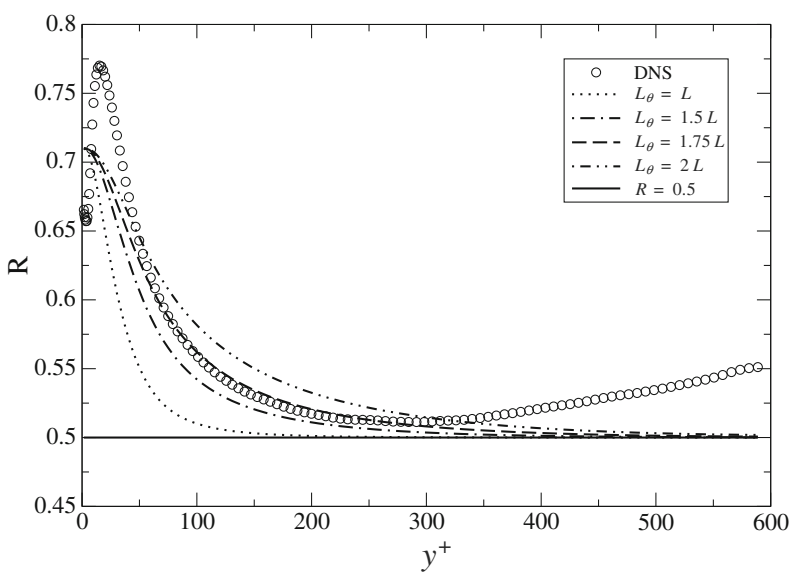

It is found in Fig. 2 that the optimal value for $L_{\theta} / L$ is 1.75 . Next section is devoted to the investigation of the role of this length scale in the modeling of $\phi_{\theta i}^{*}-\varepsilon_{\theta i}$.

\section{Influence of the Modeling of the Thermal Length Scale}

As mentioned in Section 2.2, Shin et al. [30] assumed that the blending function $\alpha_{\theta}$ used in Eq. 13 is equal to the blending function $\alpha$ used in Eq. 3, which is a strong assumption. Since $\alpha$ and $\alpha_{\theta}$ are obtained from similar elliptic Eqs. 4 and 14, this is equivalent to the assumption $L_{\theta}=L$. Actually, $L_{\theta}$ originates from the approximation of the two-point correlations by exponential functions in Eq. 11, and the correlation length scale has no reason to be the same for temperature-pressure correlations as for velocity-pressure correlations. At large turbulent Peclet numbers $P e_{t}=R e_{t} P r$, the large-scale temperature fluctuations are simply convected, such that $L_{\theta}$ can be considered entirely determined by the velocity field, and assuming a constant $L_{\theta} / L$ is reasonable, but this does not imply that the constant is exactly one. Therefore, the present section focuses on the modeling of the thermal length scale $L_{\theta}$, and its influence on the predictions of $\phi_{\theta i}^{*}$ and $\varepsilon_{\theta i}$, using a priori tests in forced and mixed convection regimes.

In order to cover a wide range of regimes, it is usual to assume that the time-scale used in heat fluxes models is a function $k, \varepsilon, k_{\theta}$ and $\varepsilon_{\theta}$, i.e., a hybrid of mechanical and thermal time-scales (see, e.g., Abe et al. [2]). This hypothesis can be extended to $L_{\theta}$, and a dimensional analysis shows that the ratio $L_{\theta} / L$ is then a function of $R$

$$
\frac{L_{\theta}}{L}=f(R),
$$

where $L=k^{3 / 2} / \varepsilon$. In order to account for the variations of $R$ in the near-wall region in a simple manner, Eq. 39 can be approximated by a linear relation $f(R)$

$$
L_{\theta}=C_{L}^{\prime} R L
$$


Moreover, as mentioned above, assuming a constant thermal-to-mechanical timescale ratio $R$ is usual for large Peclet numbers. This assumption implies a constant ratio $L_{\theta} / L$.

In order to assess a priori the validity of the simple relation 40 and to evaluate the coefficient $C_{L}^{\prime}$, two DNS databases of fully developed turbulent channel flows are used, in forced and mixed convection regimes. The a priori procedure consists in first solving Eq. 14, with the modeled $L_{\theta}$ evaluated using DNS data, and then computing the model 13, also from DNS data. The standard coefficients [23] of the model for the scrambling term Eqs. 22 and 23 are used $\left(C_{\theta 1}=3, C_{\theta 2}=0.55, C_{\theta 2}^{\prime}=0, C_{\theta 3}=0.55\right.$, $\left.C_{\theta 1}^{\prime}=0\right)$, which yields for the EB-AFM model (Eq. 34)

$$
\begin{gathered}
\xi=\left(1-0.55 \alpha_{\theta}^{3}\right), \quad \zeta=1, \quad \chi=0, \quad \eta=\left(1-0.55 \alpha_{\theta}^{3}\right), \\
\gamma=\left(1-\alpha_{\theta}^{3}\right)\left(1+C_{\varepsilon}\right), \quad C_{\varepsilon}=\frac{1}{2}\left(1+\frac{1}{P r}\right), \\
\text { and } C_{\theta}=\frac{C_{\theta}^{\prime}}{3 \alpha_{\theta}^{3}+\left(1-\alpha_{\theta}^{3}\right) C_{\varepsilon}},
\end{gathered}
$$

where $C_{\theta}^{\prime}=0.68$ is used.

In addition to case of Abe and Kawamura [1] described above and in Fig. 3, the DNS database of Kasagi and Nishimura [18] for the mixed convection regime is used. Buoyancy is accounted for in a vertical channel flow driven by a pressure gradient, with an imposed wall-temperature difference (see Fig. 4). The Reynolds number based on the mean friction velocity is $R e_{\tau}=150$. The Prandtl number is $\operatorname{Pr}=0.71$ and the Grashof number based on the temperature difference $G r=9.6 \times 10^{5}$.

Figures 5 and 6 show $\phi_{\theta 1}^{*}-\varepsilon_{\theta 1}$ (streamwise direction) and $\phi_{\theta 2}^{*}-\varepsilon_{\theta 2}$ (wall-normal direction), respectively, obtained with different values of $L_{\theta}$. Figures 7 and 8 show the same results for the mixed convection case. The difference of sensitivity to a modification of the length of these two components is striking. Contrary to $\phi_{\theta 1}^{*}-\varepsilon_{\theta 1}$, for which doubling the length scale yields a significant modification of the results, $\phi_{\theta 2}^{*}-\varepsilon_{\theta 2}$ remains nearly unaffected. The reason for this behavior lies in the term $\gamma \frac{\varepsilon}{k} \overline{u_{k}^{\prime} \theta^{\prime}} n_{i} n_{k}$ arising when the elliptic blending approach is introduced (see Eq. 34). Indeed, the normal-wise component writes

$$
\overline{v^{\prime} \theta^{\prime}}=-C_{\theta} \frac{k}{\varepsilon}\left(\overline{v^{\prime 2}} \frac{\partial \bar{\theta}}{\partial y}+\gamma \frac{\varepsilon}{k} \overline{v^{\prime} \theta^{\prime}}\right),
$$

Fig. 3 Channel flow in forced convection regime, Abe and Kawamura [1] $\dot{q}_{w}$

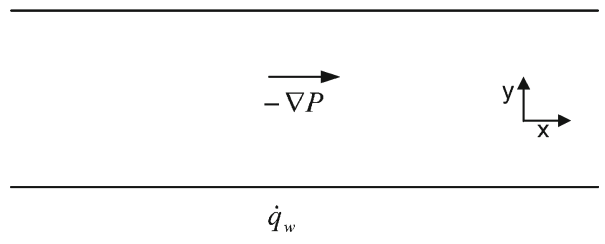


Fig. 4 Channel flow in mixed convection regime, Kasagi and Nishimura [18]

which can be recast as

$$
\overline{v^{\prime} \theta^{\prime}}=-C_{\theta}^{*} \frac{k}{\varepsilon}\left(\overline{v^{\prime 2}} \frac{\partial \bar{\theta}}{\partial y}\right)
$$

where

$$
C_{\theta}^{*}=\frac{C_{\theta}^{\prime}}{C_{\theta 1}}\left[\alpha_{\theta}^{3}+\left(1-\alpha_{\theta}^{3}\right) \frac{C_{\varepsilon}+\left(1+C_{\varepsilon}\right) C_{\theta}^{\prime}}{C_{\theta 1}}\right]^{-1} .
$$

Far from the wall, $C_{\theta}^{*} \rightarrow C_{\theta}^{\prime} / C_{\theta 1}$, and Eq. 42 tends to the form given by standard models (GGDH or AFM).

For $\operatorname{Pr}=0.71, C_{\varepsilon} \approx 1.204$, such that $C_{\theta}^{*}$ goes from 0.23 far from the wall to 0.25 at the wall. Therefore, whatever the shape of $\alpha_{\theta}$, i.e., whatever the model used for $L_{\theta}, \overline{v^{\prime} \theta^{\prime}}$ remains nearly the same. This remark leads to an important conclusion: the reason why standard models (GGDH and AFM) successfully reproduce the mean

Fig. 5 A priori test of the model for $\phi_{\theta 1}^{*}-\varepsilon_{\theta 1}$ in forced convection regime [1] $\left(R e_{\tau}=640\right)$

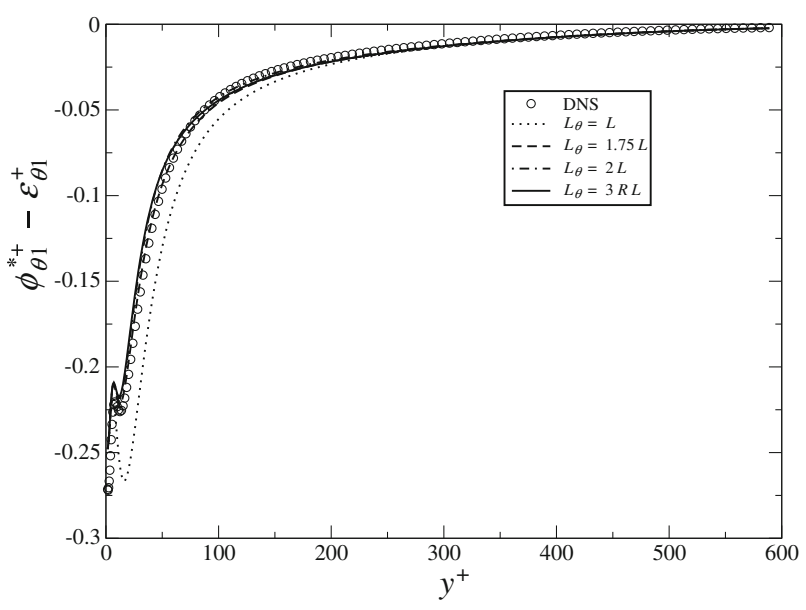


Fig. 6 Same figure as Fig. 5 for $\phi_{\theta 2}^{*}-\varepsilon_{\theta 2}$

Fig. 7 A priori test of the model for $\phi_{\theta 1}^{*}-\varepsilon_{\theta 1}$ in mixed convection regime [18]

Fig. 8 Same figure as Fig. 7 for $\phi_{\theta 2}^{*}-\varepsilon_{\theta 2}$
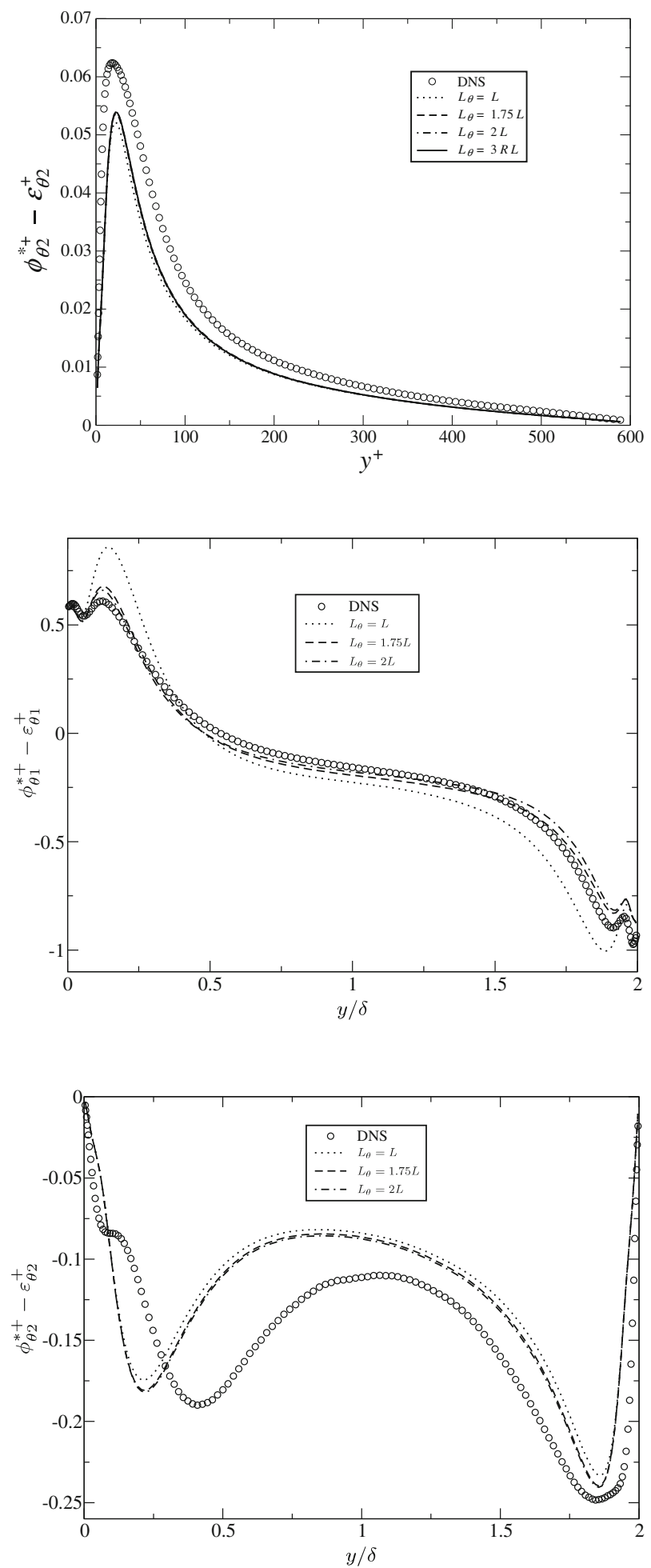
Fig. $9 C_{\theta}^{*}$ sensitivity to the Prandtl number

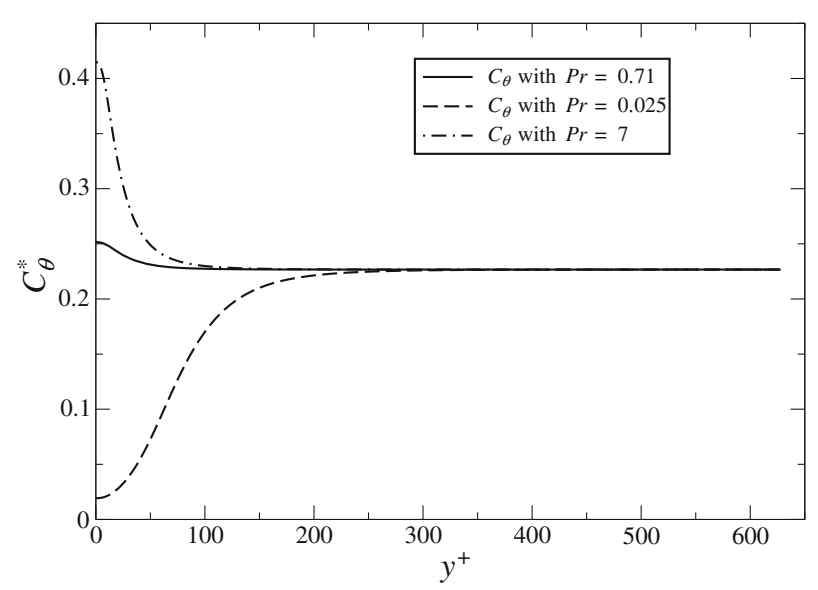

temperature profile in channel flows for fluids with a Prandtl number close to unity, despite the fact that they do not account for near-wall effects on the heat fluxes, is a coincidence. Indeed, in such flows, the streamwise heat flux is not active, and the wall-normal heat flux is correctly reproduced because, for such Prandlt numbers, $C_{\theta}^{*}$ is almost a constant. Of course, this feature relies on the correct prediction of the wall-normal component of the Reynolds stress, for which accounting for near-wall effects (which is the case with the EB-RSM) is crucial. However, the situation is different in more complex geometries, where a correct prediction of all the component of the turbulent heat fluxes is important, as well as for Prandtl numbers far from unity: Fig. 9 illustrates the distribution of the coefficient $C_{\theta}^{*}$ in the domain for different values of the Prandtl number. It is clearly seen that the variation of $C_{\theta}^{*}$ cannot be neglected close to the wall for large or small Prandtl numbers, and, consequently, accounting for wall effects in the model for the turbulent heat fluxes is necessary to correctly reproduce temperature profiles. It is worth pointing out that, for such values of the Prandtl number, other modeling issues arise, such as the modeling of $\varepsilon_{\theta i}^{h}$, which cannot be neglected for small Prandtl numbers, since the small-scale temperature fluctuations can be considered isotropic only at the limit of large Peclet numbers [21,34].

On the contrary, for both cases, the streamwise component $\phi_{\theta 1}^{*}-\varepsilon_{\theta 1}$ is significantly improved by increasing the ratio $L_{\theta} / L$ (albeit not shown here, the improvement is particularly pronounced for $\phi_{\theta 1}^{*}$ ). Considering the present a priori test and the conclusion of Section 3.3, the optimal value is $L_{\theta} / L=1.75$. In the forced convection case, since the time-scale ratio $R$ is provided by the database, the linear hypothesis (Eq. 40) can be also evaluated with a variable $R$. It can be observed that the results obtained with $L_{\theta}=3 R L$ and $L_{\theta}=1.75 L$ are almost identical, such that using a constant ratio $L_{\theta} / L$ is considered sufficient.

\section{Computational Results}

EDF in-house open source (http://www.code-saturne.org) CFD tools Code_Saturne is used for the present computations. Code_Saturne is an unstructured, collocated 
finite volume solver for cells of any shape. Spatial discretization is second order accurate. The Navier-Stokes equations are solved for turbulent incompressible flows using a SIMPLEC algorithm for pressure-velocity coupling (see Archambeau et al. [3] for additional details about the code). It includes several RANS models with first and second moment closures such as the standard $k-\varepsilon, k-\omega$-SST, stabilized $\overline{v^{2}}-f(\phi$ model), LRR and SSG models. The code can also perform Large-Eddy Simulation using standard or dynamic Smagorinsky models. The elliptic blending approach has been introduced into the standard SSG model available in Code_Saturne in order to implement the EB-RSM, which is used for all the computations. For all the solutions presented in the present paper, grid convergence has been carefully ensured. The first computational point for both test cases are located around $y^{+}=0.1$.

The different turbulent heat fluxes models used in the present work, and summarized below, have been implemented:

- GGDH (Generalized Gradient Diffusion Hypothesis)

$$
\overline{u_{i}^{\prime} \theta^{\prime}}=-C_{\theta} \frac{k}{\varepsilon} \overline{u_{i}^{\prime} u_{j}^{\prime}} \frac{\partial \bar{\theta}}{\partial x_{j}}
$$

with $C_{\theta}=0.235$.

- AFM (Algebraic Flux Model)

$$
\overline{u_{i}^{\prime} \theta^{\prime}}=-C_{\theta} \frac{k}{\varepsilon}\left[\overline{u_{i}^{\prime} u_{j}^{\prime}} \frac{\partial \bar{\theta}}{\partial x_{j}}+\xi \overline{u_{j}^{\prime} \theta^{\prime}} \frac{\partial \overline{u_{i}}}{\partial x_{j}}+\eta \beta g_{i} \overline{\theta^{\prime 2}}\right]
$$

with $C_{\theta}=0.235, \xi=0.45$ and $\eta=0.45$.

- $\quad$ EB-GGDH (Elliptic Blending-Generalized Gradient Diffusion Hypothesis)

$$
\overline{u_{i}^{\prime} \theta^{\prime}}=-C_{\theta} \frac{k}{\varepsilon}\left[\overline{u_{i}^{\prime} u_{j}^{\prime}} \frac{\partial \bar{\theta}}{\partial x_{j}}+\gamma \frac{\varepsilon}{k} \overline{u_{k}^{\prime} \theta^{\prime}} n_{i} n_{k}\right]
$$

with $C_{\theta}=\frac{0.68}{3 \alpha_{\theta}^{3}+\left(1-\alpha_{\theta}^{3}\right) C_{\varepsilon}}, \gamma=\left(1-\alpha_{\theta}^{3}\right)\left[1+C_{\varepsilon}\right]$ and $C_{\varepsilon}=\frac{1}{2}\left(1+\frac{1}{\operatorname{Pr}}\right)$

- $\quad$ The EB-AFM (Elliptic Blending-Algebraic Flux Model)

$$
\overline{u_{i}^{\prime} \theta^{\prime}}=-C_{\theta} \frac{k}{\varepsilon}\left[\overline{u_{i}^{\prime} u_{j}^{\prime}} \frac{\partial \bar{\theta}}{\partial x_{j}}+\xi \overline{u_{j}^{\prime} \theta^{\prime}} \frac{\partial \overline{u_{i}}}{\partial x_{j}}+\eta \beta g_{i} \overline{\theta^{\prime 2}}+\gamma \frac{\varepsilon}{k} \overline{u_{j}^{\prime} \theta^{\prime}} n_{i} n_{j}\right]
$$

with $\xi=1-0.55 \alpha_{\theta}^{3}$ and $\eta=1-0.55 \alpha_{\theta}^{3}$ (these coefficients tend to the coefficients of the AFM far from the wall). The temperature variance $\overline{\theta^{\prime 2}}$ is obtain with the Eqs. 37 and 38 .

It is worth pointing out that the coefficients used in the GGDH, AFM, EB-GGDH and EB-AFM all originate from the same calibration [23] of the underlying model for the scrambling term (Eqs. 22 and 23): $C_{\theta 1}=3, C_{\theta 2}=0.55, C_{\theta 2}^{\prime}=0, C_{\theta 3}=0.55$, $C_{\theta 1}^{\prime}=0$. The recalibration coefficient (see the discussion in Section 3.1) $C_{\theta}^{\prime}$, involved in $C_{\theta}=C_{\theta}^{\prime} / C_{\theta 1}$ is chosen for each model in order to optimize the reproduction of the mean temperature profile in the forced convection case. The corresponding values are $C_{\theta}^{\prime}=0.705$ for GGDH and AFM, and $C_{\theta}^{\prime}=0.68$ for EB-GGDH and EB-AFM. 
Fig. 10 The velocity profile (computational results for the forced convection regime, $\left.R e_{\tau}=640\right)$

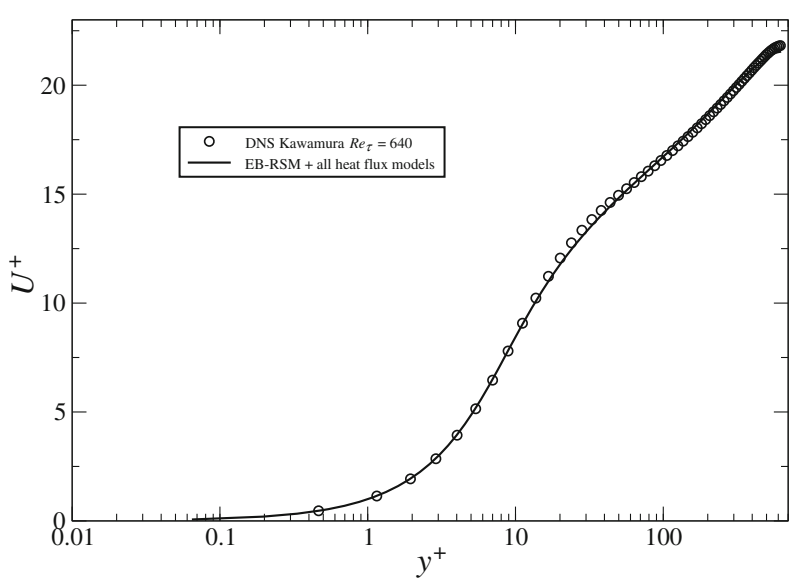

Computations were performed in order to evaluate the advantage of the introduction of the elliptic blending approach combined either with the GGDH or the AFM model on the prediction of the turbulent heat fluxes and mean velocity/temperature profiles, as well as to confirm the necessity of using a thermal length scale different from the mechanical length scale. The two DNS test-cases used above for a priori tests are investigated.

Forced convection regime In a fully developed turbulent channel flow, the EB-RSM model gives very satisfactory results for the mean velocity and the Reynolds stresses, as shown in Fig. 10 and in Fig. 11, but this is of course independent of the turbulent heat fluxes model. Figure 12 shows the temperature profile for the different turbulent heat fluxes models. Note that, in forced convection, the AFM and EB-AFM results give exactly the same results as the GGDH or the EB-GGDH, respectively. As mentioned in Section 4, the accounting for the near-wall effects only weakly modifies the prediction of the mean temperature profile, since the wall-normal heat flux is only marginally affected by the elliptic blending approach. This is confirmed by Fig. 13, in

Fig. 11 The Reynolds tensor profile. (Symbols: DNS of Kawamura $R e_{\tau}=640$ [1]) (computational results for the forced convection regime)

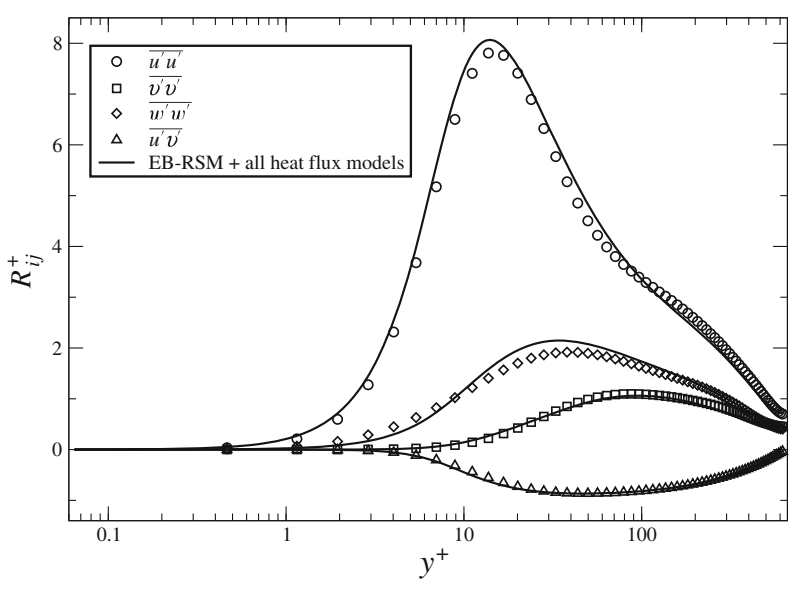


Fig. 12 The temperature profile (computational results for the forced convection regime, $R e_{\tau}=640$ )

Fig. 13 The normal-wise turbulent heat flux $\overline{v^{\prime} \theta^{\prime}}$ (computational results for the forced convection regime, $R e_{\tau}=640$ )

Fig. 14 The streamwise turbulent heat flux $\overline{u^{\prime} \theta^{\prime}}$ with GGDH and EB-GGDH flux models (computational results for the forced convection regime, $R e_{\tau}=640$ )
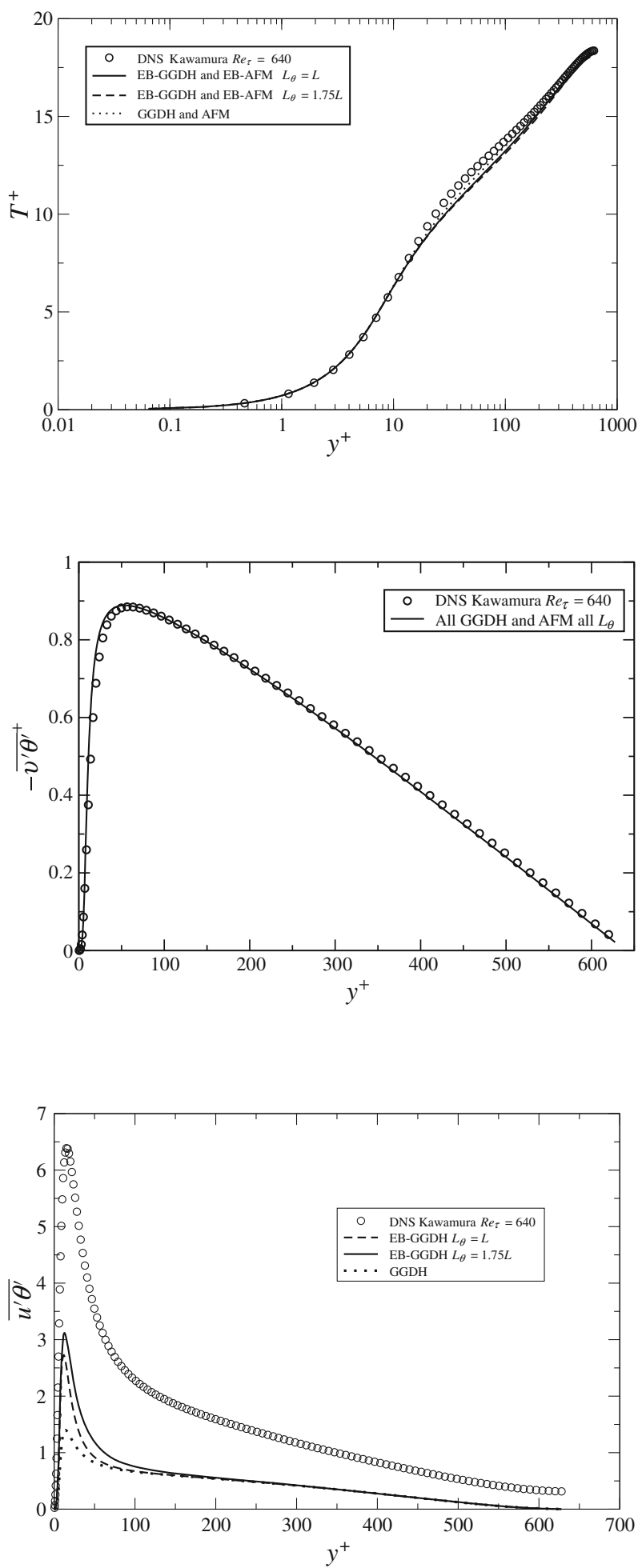
Fig. 15 The streamwise turbulent heat flux $\overline{u^{\prime} \theta^{\prime}}$ with AFM and EB-AFM flux models (computational results for the forced convection regime, $R e_{\tau}=640$ )

Fig. 16 The velocity profile (computational results for the mixed convection regime, $\left.R e_{\tau}=150, G r=9.6 \times 10^{5}\right)$

Fig. 17 The Reynolds tensor profile. (Symbols: DNS of Kasagi [18]) (computational results for the mixed convection regime)
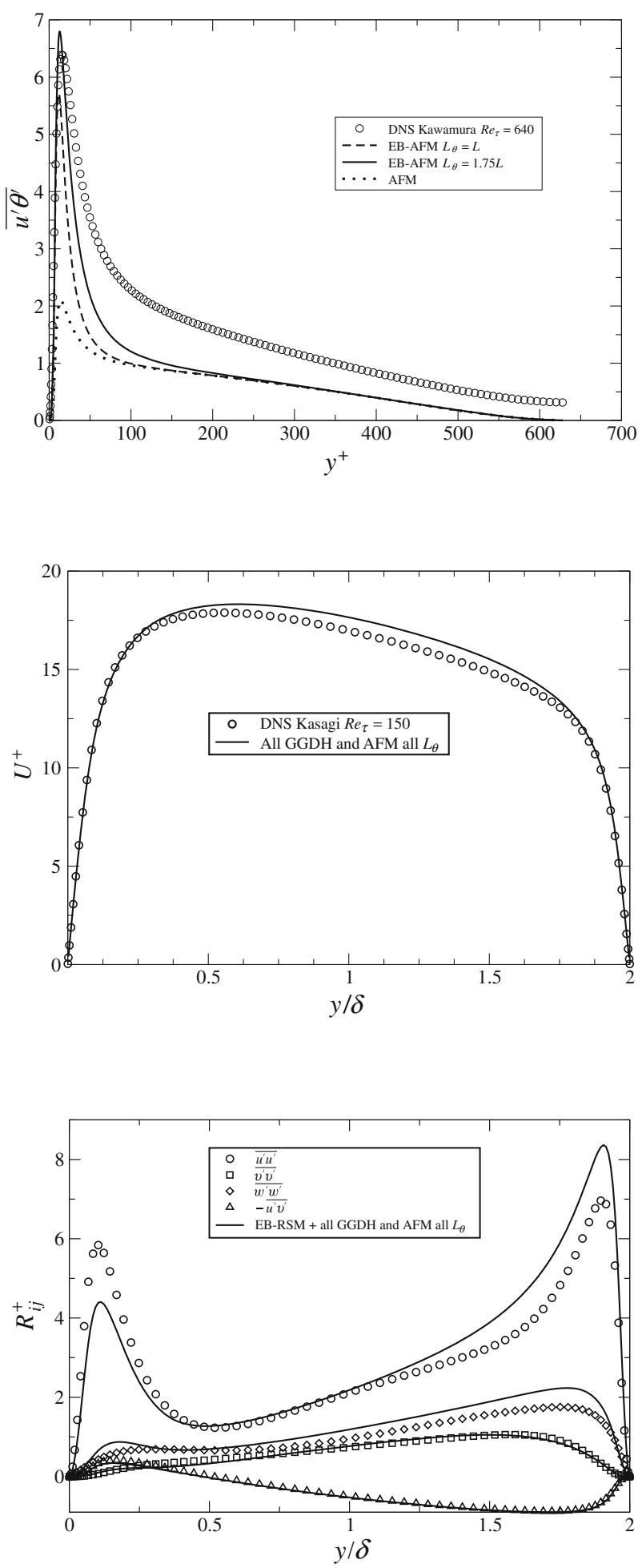
Fig. 18 The temperature profile (computational results for the mixed convection regime, $R e_{\tau}=150$, $G r=9.6 \times 10^{5}$ )

Fig. 19 Wall normal heat flux $\overline{v^{\prime} \theta^{\prime}}$ (computational results for the mixed convection regime, $R e_{\tau}=150, G r=9.6 \times 10^{5}$ )

Fig. 20 streamwise heat flux $\overline{u^{\prime} \theta^{\prime}}$ (computational results for the mixed convection regime, $R e_{\tau}=150, G r=9.6 \times 10^{5}$ )
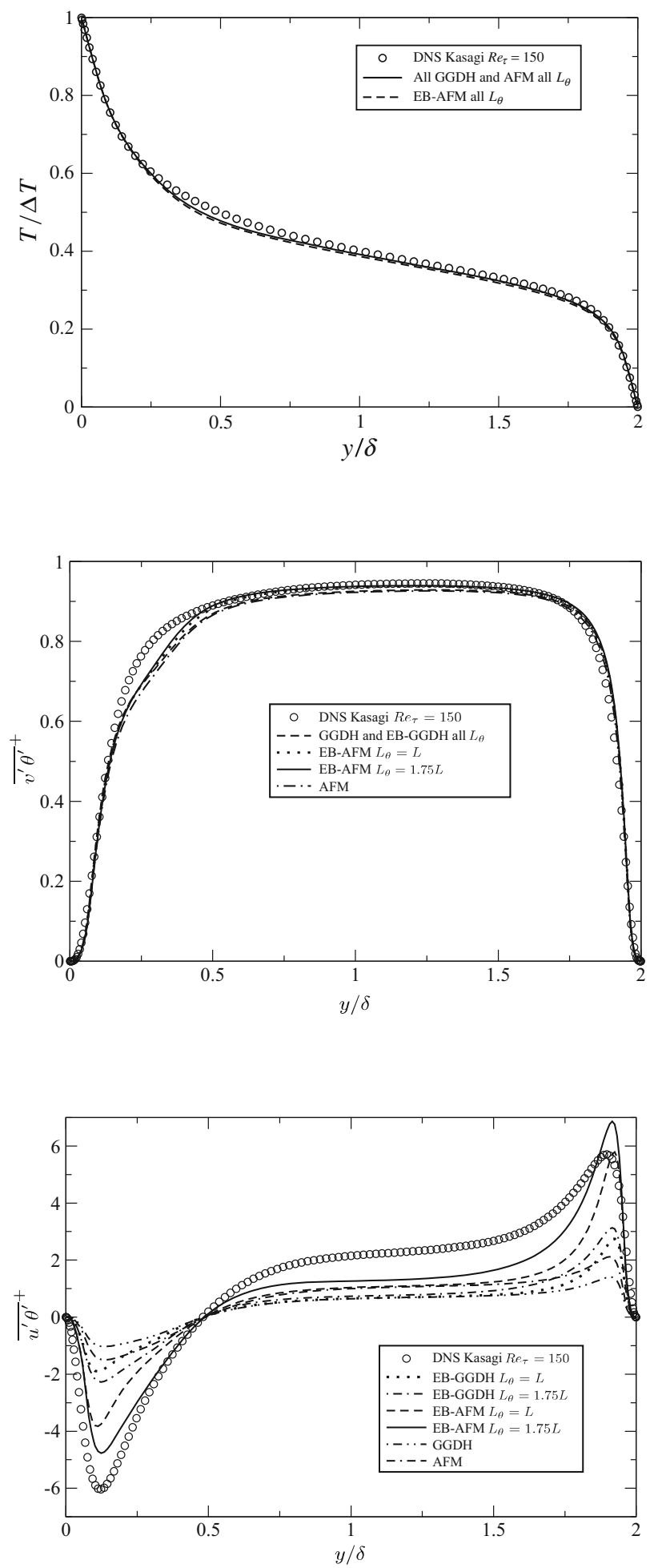
Fig. 21 Temperature variance $\overline{\theta^{\prime 2}}$ (computational results for the mixed convection regime, $R e_{\tau}=150, G r=9.6 \times 10^{5}$ )

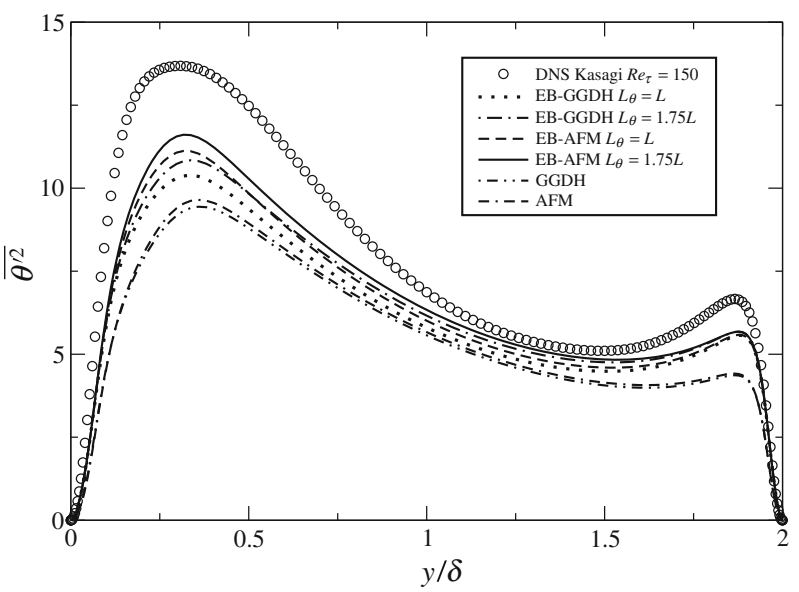

which it can be seen that the predictions given by all the heat fluxes models are not distinguishable.

For the sake of clarity, the predictions for the streamwise heat flux by the GGDH and AFM models have been split in the two Figs. 14 and 15. These two figures clearly show the improvement in the near-wall region introduced by elliptic blending, in particular in the case of the AFM. Moreover, the benefit of using a thermal length scale different from the mechanical length scale is confirmed, although it does not constitute a major breakthrough. It is worth pointing out that differentiating these two length scales has a computational cost, since it makes necessary the resolution of an additional elliptic equation for $\alpha_{\theta}$ (otherwise $\alpha_{\theta}=\alpha$ ), but the cost of the resolution of this equation is very low (typically less than $1 \%$ of the total computational time).

Mixed convection For the mixed convection regime, the EB-RSM gives satisfactory results for the mean velocity (Fig. 16) and the Reynolds Tensor (Fig. 17) whatever the heat fluxes model. Contrary to the case of forced convection, it can be seen in Figs. 18 and 19 that the predictions of the wall-normal heat flux and, consequently, the mean temperature, are slightly influenced by the introduction of the near-wall effects. The most significant improvement is again for the streamwise component (Fig. 20), but also for the temperature variance, as seen in Fig. 21. Overall, the value $L_{\theta} / L=1.75$ is an appropriate choice, which confirms the conclusion of Section 3.3.

\section{Conclusions}

The main contribution of the present paper is the proposal of a new algebraic model for the turbulent heat fluxes, the EB-AFM, as well as a simplified form, the EBGGDH. These models are similar to usual AFM and GGDH, but reproduce the influence of the wall on the heat fluxes. They are algebraic versions of the EB-DFM [30], based on transport equations for the turbulent heat fluxes in which the blocking effect of the wall on the scrambling term is reproduced by the elliptic blending approach (hence the EB prefix). 
Moreover, the analytical justification of the use of the elliptic relaxation approach for the turbulent heat fluxes was introduced, which forms the basis for the EB-DFM. In addition, it was proposed to refine the modeling of the thermal-to-mechanical time-scale ratio $R$, used to evaluate the dissipation of the temperature variance, in order to impose the correct wall limit, as well as to distinguish the mechanical and thermal length scales in the elliptic blending approach for the Reynolds stresses and the heat fluxes, respectively.

The validation of these proposals was carried out using both a priori tests and numerical simulations in forced and mixed convection regimes. The predictions of the streamwise turbulent heat flux and the temperature variance are significantly improved when the near-wall effects are accounted for by elliptic blending. Using a thermal length scale larger than the mechanical length scale proved also beneficial, although the improvement is moderate. On the contrary, in channel flow cases, for fluids with Prandtl numbers close to unity, the wall-normal turbulent heat flux is only moderately affected by the introduction of the effects of the wall, which provides an explanation for the success of standard models (AFM and GGDH) in channel flow configurations, although they derive from a model not valid in the near-wall region. This is to be traced to the fact that, fortuitously, the two limiting values (at the wall and far from the wall) of the variable coefficient driving this component of the heat fluxes are almost equal for $\operatorname{Pr}=0.71$. Accounting for the near-wall effects might have an important influence in cases where the Prandtl number is far from unity.

\section{References}

1. Abe, H., Kawamura, H., Matsuo, Y.: Surface heat-flux fluctuations in a turbulent channel flow up to $\operatorname{Re}_{\tau}=1,020$ with $\operatorname{Pr}=0.025$ and 0.71. Int. J. Heat Fluid Flow 25, 404-419 (2004)

2. Abe, K., Kondoh, T., Nagano, Y.: A new turbulence model for predicting fluid flow and heat transfer in separating and reattaching flows-II. Thermal field calculations. Int. J. Heat Mass Transfer 38(8), 1467-1481 (1995)

3. Archambeau, F., Méchitoua, N., Sakiz, M.: Code Saturne: a finite volume code for the computation of turbulent incompressible flows-industrial applications. Int. J. Finite Vol. 1(1), 1-62 (2004). ISSN 1634(0655) URL: http://averoes.math.univ-paris13.fr/html

4. Borello, D., Hanjalic, K., Rispoli, F.: Prediction of cascade flows with innovative second-moment closures. J. Fluids Eng.-T. ASME 127(6), 1059-1070 (2005)

5. Dol, H.S., Hanjalic̀, K., Kenjereš, S.: A comparative assessment in the second-moment differential and algebraic models in turbulent natural convection. Int. J. Heat Fluid Flow 18, 4-14 (1997)

6. Durbin, P.A.: Near-wall turbulence closure modeling without "damping functions". Theor. Comput. Fluid Dyn. 3, 1-13 (1991)

7. Durbin, P.A.: A Reynolds stress model for near-wall turbulence. J. Fluid Mech. 249, 465-498 (1993)

8. Durbin, P.A.: Limiters and wall treatments in applied turbulence modeling. Fluid Dyn. Res. 41(012203) (2009). doi:10.1088/0169-5983/41/1/012203

9. Fadai-Ghotbi, A., Friess, C., Manceau, R., Borée, J.: A seamless hybrid RANS-LES model based on transport equations for the subgrid stresses and elliptic blending. Phys. Fluids 22(055104) (2010). doi:10.1063/1.3415254

10. Fadai-Ghotbi, A., Friess, C., Manceau, R., Gatski, T., Borée, J.: Temporal filtering: a consistent formalism for seamless hybrid RANS-LES modeling in inhomogeneous turbulence. Int. J. Heat Fluid Flow 31(3), 378-389 (2010)

11. Fadai-Ghotbi, A., Manceau, R., Borée, J.: Revisiting URANS computations of the backwardfacing step flow using second moment closures. Influence of the numerics. Flow Turbulence Combust. 81(3), 395-414 (2008) 
12. Gatski, T.B., Rumsey, C.L., Manceau, R.: Current trends in modeling research for turbulent aerodynamic flows. Philos. Trans. R. Soc. A 365(1859), 2389-2418 (2007)

13. Han, S., Choi, Y., Shin, J., Kim, Y., Kim, M.: Turbulent heat transfer of supercritical carbon dioxide in square cross-sectional duct flow. J. Mech. Sci. Technol. 22(12), 2563-2577 (2008)

14. Hanjalić, K.: One-point closure models for buoyancy-driven turbulent flows. Annu. Rev. Fluid Mech. 34, 321-347 (2002)

15. Hanjalić, K., Kenjereš, S., Durst, F.: Natural convection in partitioned two-dimensional enclosures at higher Rayleigh numbers. Int. J. Heat Mass Transfer 39(7), 1407-1427 (1996)

16. Ho, H., Don, C., Sik, S., Kun, S.: Compressibility factor effect on the turbulence heat transfer of super-critical carbon dioxide by an elliptic-blending second moment closure. Trans. Korean Soc. Mech. Engineers B 31(1), 40-50 (2007)

17. Ince, N.Z., Launder, B.: On the computation of buoyancy-driven turbulent flows in rectangular enclosures. Int. J. Heat Fluid Flow 10(2), 110-117 (1989)

18. Kasagi, N., Nishimura, M.: Direct numerical simulation of combined forced and natural turbulent convection in a vertical plane channel. Int. J. Heat Fluid Flow 18(1), 88-99 (1997)

19. Kenjereš, S., Gunarjo, S., Hanjalić, K.: Contribution to elliptic relaxation modelling of turbulent natural and mixed convection. Int. J. Heat Fluid Flow 26(4), 569-586 (2005)

20. Kenjereš, S., Hanjalić, K.: Prediction of turbulent thermal convection in concentric and eccentric horizontal annuli. Int. J. Heat Fluid Flow 16(5), 429-439 (1995)

21. Kenjereš, S., Hanjalic̀, K.: Numerical simulation of magnetic control of heat transfer in thermal convection. Int. J. Heat Fluid Flow 25, 559-568 (2004)

22. Launder, B.E.: On the effects of a gravitational field on the turbulent transport of heat and momentum. J. Fluid Mech. 67(3), 569-581 (1975)

23. Launder, B.E.: On the computation of convective heat transfer in complex turbulent flows. J. Heat Transfer 110, 1112-1128 (1988)

24. Manceau, R.: An improved version of the elliptic blending model. Application to non-rotating and rotating channel flows. In: Proc. 4th Int. Symp. Turb. Shear Flow Phenomena. Williamsburg, VA (2005)

25. Manceau, R., Hanjalić, K.: Elliptic blending model: a new near-wall Reynolds-stress turbulence closure. Phys. Fluids 14(2), 744-754 (2002)

26. Manceau, R., Wang, M., Laurence, D.: Inhomogeneity and anisotropy effects on the redistribution term in Reynolds-averaged Navier-Stokes modelling. J. Fluid Mech. 438, 307-338 (2001)

27. Oceni, A.G., Manceau, R., Gatski, T.: Introduction of wall effects in explicit algebraic stress models through elliptic blending. In: Stanislas, M., Jimenez, J., Marusic, I. (eds.) Progress in wall turbulence: Understanding and Modelling. Springer (2010)

28. Rotta, J.C.: Statistische theorie nichthomogener turbulenz. Z. Phys. 129(6), 547-572 (1951)

29. Shin, J.: Modeling of turbulent heat transfer in an axially rotating pipe flow. Trans. Korean Soc. Mech. Engineers B 31(9), 741-753 (2007)

30. Shin, J.K., An, J.S., Choi, Y.D., Kim, Y.C., Kim, M.S.: Elliptic relaxation second moment closure for the turbulent heat fluxes. J. Turbul. 9(3), 1-29 (2008)

31. Shin, J.K., Choi, Y.D., An, J.S.: Numerical analysis of turbulent flow and heat transfer in a square sectioned U-bend duct by elliptic-blending second moment closure. Journal of Mechanical Science and Technology 21(2), 360-371 (2007)

32. Shin, J.K., Chun, K.H., Choi, Y.D.: Refinement of a second moment closure by the elliptic blending equation and its application to turbulent rotating channel flows. J. Turbul. 4(030), 1-22 (2003)

33. Speziale, C.G., Sarkar, S., Gatski, T.B.: Modeling the pressure-strain correlation of turbulence: an invariant dynamical system approach. J. Fluid Mech. 227, 245-272 (1991)

34. Tennekes, H., Lumley, J.L.: A First course in Turbulence. MIT Press (1972)

35. Thielen, L., Hanjalić, K., Jonker, H., Manceau, R.: Predictions of flow and heat transfer in multiple impinging jets with an elliptic-blending second-moment closure. Int. J. Heat Mass Transfer 48(8), 1583-1598 (2005)

36. Törnblom, O., Johansson, A.V.: A Reynolds stress closure description of separation control with vortex generators in a plane asymmetric diffuser. Phys. Fluids 19(11), 115108-115115 (2007)

37. Viti, V., Huang, G., Bradshaw, P.: Numerical study of stress-transport turbulence models: Implementation and validation issues. Comput. Fluids 36(8), 1373-1383 (2007) 